and growth dysfunction, and cognitive impairments. Basal-cell carcinoma, discussed below, is an example of a secondary malignancy induced by spinal irradiation for medulloblastoma. Low risk tumors, identified by the biological method, may be spared unnecessary aggressive and disabling therapy.

\title{
NEVOID BASAL-CELL CARCINOMA (GORLIN) SYNDROME
}

A unique case of a 15-year-old girl with nevoid basal-cell carcinoma (NBCCS), who presented with uncontrolled temporal lobe epilepsy due to neuronal heterotopia, is reported from the Royal Melbourne Hospital, Australia. Seizures began at age 13 months, often precipitated by fever. She had a dermoid cyst removed from her nose, multiple dental cysts, and seborrheic keratosis of the scalp. Growth increased dramatically at age 9 years, and seizures became refractory to treatment. Chest X-ray showed cervical spina bifida occulta and fused ribs. Head CT showed calcification of falx cerebri and tentorium. MRI showed a lesion in the left anterior temporal lobe. EEG recorded seizure activity in the left frontotemporal areas. Temporal lobectomy specimen showed neuronal heterotopias and cortical dysplasia. After surgery, she was seizure free at 6 months follow-up. (Hogan RE et al. Epilepsy in the nevoid basal-cell carcinoma syndrome (Gorlin syndrome): Report of a case due to a focal neuronal heterotopia. Neurology Feb 1996;46:574-576). (Reprints: Dr R Edward Hogan, Department of Neurology, St Louis University, 3635 Vista Ave at Grand Blvd, PO Box 15250, St Louis, MO 63110).

COMMENT. Nevoid basal-cell carcinoma syndrome presents at puberty with multiple basal-cell carcinomata, palmar and plantar pits, odontogenic painful cysts of the mandible, facial paresthesia, macrocephaly, large stature, frontal bossing, broad nasal bridge, narrow shoulders, fused ribs, polydactyly, bone cysts, calcified falx, cervical spina bifida occulta, odontoid agenesis, corpus callosum agenesis, meningioma, and medulloblastoma. Medulloblastoma, occurring in 3-5\% of cases, may precede the development of other manifestations of the syndrome, and may have a better prognosis than general. Patients with seizures should be investigated for neuronal heterotopias, which may be treated surgically. The syndrome is autosomal dominant and the gene is localized to chromosome $9 q 22$.

\section{INTRACRANIAL GERM-CELL TUMORS}

Prognosis of 26 children with intracranial germ-cell tumors (17 germinomas and 9 teratomas) treated in a 10 year period is reported from the University Hospital Hamburg-Eppendorf, Germany. Median age at diagnosis was 11.5 years (range 1.5-16.8 years). Tumor location was in the pineal region in $69 \%$ and suprasellar/hypothalamic in 31\%. Symptoms were increased intracranial pressure, Parinaud's syndrome, and endocrine deficits. Surgical resection was attempted in 22 patients, 25 were irradiated, and 8 received additional chemotherapy. Long-term survival rate was $88 \%$ for germinomas and $43 \%$ for malignant teratomas; $57 \%$ had no and $37 \%$ had mild neurologic deficits, and $24 \%$ had neuro-endocrine dysfunction. Neuropsychological function was normal or only mildly impaired in $53 \%$, and $69 \%$ had no change in their level of education. (Haupt C et al. Intracranial germ-cell tumours treatment results and residuals. Eur I Pediatr March 1996;155:230-236). (Respond: Dr C Haupt, Departments of Paediatrics and Neurosurgery, University Hospital HamburgEppendorf, D-20251 Hamburg, Germany).

COMMENT. In this center, primary intracranial germ-cell tumors (PGCT) 
accounted for $8.5 \%$ of childhood brain tumors, while other series report a frequency of less than $5 \%$. Germinomas arising in the pineal or suprasellar region are the most common form (65\%) of PGCT and have a good prognosis, with $88 \%$ long-term survival after surgical removal and radiation. Severe residual complications, including cognitive deficits, are uncommon, despite post-operative craniospinal axis irradiation. Malignant teratomas have a less favorable prognosis. The younger age at which medulloblastomas are treated with irradiation accounts for the higher incidence of cognitive deficits in these tumor patients.

\section{HYPOXIC SYNDROMES}

\section{BREATH-HOLDING SPELLS}

The clinical characteristics, types, diagnosis, and management of breath-holding spells are reviewed from The Department of Pediatrics (Neurology), Park Nicollet Medical Center, Minneapolis, MN. There are two forms, pallid and cyanotic. Pallid breath-holding spells (BHS) result from vagal hyperresponsiveness, following a sudden, unexpected, unpleasant stimulus, usually a mild head injury. Cardiac monitoring reveals prolonged asystoles, which can also be induced by ocular compression, and is accompanied by syncope or an anoxic seizure. Vagal cardiac inhibition with cerebral anoxia is the pathophysiology of pallid BHS. Cyanotic breath-holding spells result from a complex interplay of hyperventilation followed by apnea in expiration, and increased intrathoracic pressure. Whereas pallid BHS occur after injury, cyanotic BHS are precipitated by anger. Diagnosis may be confirmed by EEG with ocular compression and cardiac monitoring. A pallid spell is associated with cardiac asystole and EEG hypersynchronous slowing. Cyanotic spells have similar EEG changes without bradycardia or asystole. Prolonged QT syndrome is a rare but serious cause of anoxic seizure, induced by exercise, injury, or fright. Cerebral hypoxia may result from ventricular tachycardia. More protracted loss of consciousness with hypotension may indicate a cardiac pathology. A more protracted seizure following a BHS may represent an anoxic-epilepsy, requiring anticonvulsant therapy. Spontaneous remission of BHSs is to be expected, but parents require frequent reassurance about the benign nature of the spells. (Breningstall GN. Breath-holding spells. Pediatr Neurol Feb 1996;14:91-97). (Respond: Dr Breningstall, Pediatric Subspecialties, Park Nicollet Medical Center, 910 East 26th St, Suite 325, Minneapolis, MN 55404).

COMMENT. Although anticonvulsant therapy is sometimes advisable when the convulsive episode is prolonged and represents an anoxic-epilepsy, traditional therapy will not generally prevent the breath-holding spell. Perhaps some of the newer antiepileptic medications should be tried in children with numerous attacks. The therapeutic nihilistic approach to BHS practiced by many physicians is often difficult for a parent to accept. An iron deficiency anemia may be an underlying causative factor in about 20\% of cases of breath-holding (Holowach J, Thurston DL. N Eng I Med 1963;268:21). Neurologic deficits with iron deficiency anemia are discussed in Progress in Pediatric Neurology 1991, PNB Publ, pp397-8.

Apnea and bradycardia during epileptic seizures were studied at the Telemetry Unit, National Hospital for Neurology and Neurosurgery, Queen Square, London. (Nashef L et al. I Neurol Neurosurg Psychiatry March 